\title{
Blood and tissue neuroendocrine tumor gene cluster analysis correlate, define hallmarks and predict disease status
}

\author{
Mark Kidd, Ignat Drozdov and Irvin Modlin
}

Wren Laboratories, 35 NE Industrial Road, Branford, Connecticut 06405, USA

Correspondence should be addressed to I Modlin

Email

imodlin@optonline.net

\begin{abstract}
A multianalyte algorithmic assay (MAAA) identifies circulating neuroendocrine tumor (NET) transcripts $(n=51)$ with a sensitivity/specificity of $98 \% / 97 \%$. We evaluated whether blood measurements correlated with tumor tissue transcript analysis. The latter were segregated into gene clusters (GC) that defined clinical 'hallmarks' of neoplasia. A MAAA/cluster integrated algorithm (CIA) was developed as a predictive activity index to define tumor behavior and outcome. We evaluated three groups. Group 1: publically available NET transcriptome databases ( $n=15$; GeneProfiler). Group 2: prospectively collected tumors and matched blood samples ( $n=22$; qRT-PCR). Group 3: prospective clinical blood samples, $n=159$ : stable disease (SD): $n=111$ and progressive disease (PD): $n=48$. Regulatory network analysis, linear modeling, principal component analysis (PCA), and receiver operating characteristic analyses were used to delineate neoplasia 'hallmarks' and assess GC predictive utility. Our results demonstrated: group 1: NET transcriptomes identified (92\%) genes elevated. Group 2: $98 \%$ genes elevated by qPCR (fold change $>2, P<0.05$ ). Correlation analysis of matched blood/tumor was highly significant $\left(R^{2}=0.7, P<0.0001\right)$, and $58 \%$ of genes defined nine omic clusters (SSTRome, proliferome, signalome, metabolome, secretome, epigenome, plurome, and apoptome). Group 3: six clusters (SSTRome, proliferome, metabolome, secretome, epigenome, and plurome) differentiated SD from PD (area under the curve $(A U C)=0.81$ ). Integration with blood-algorithm amplified the AUC to $0.92 \pm 0.02$ for differentiating PD and SD. The CIA defined a significantly lower SD score (34.1 \pm $2.6 \%)$ than in PD $(84 \pm 2.8 \%, P<0.0001)$. In conclusion, circulating transcripts measurements reflect NET tissue values. Integration of biologically relevant GC differentiate SD from PD. Combination of GC data with the blood-algorithm predicted disease status in $>92 \%$. Blood transcript measurement predicts NET activity.
\end{abstract}

Key Words
- algorithm
- biomarker
- carcinoid
- gastroenteropancreatic
- hallmarks
- Ki-67
- multigene transcript
- neuroendocrine
- NET
- PCR
- proliferation

Endocrine-Related Cancer (2015) 22, 561-575

\section{Introduction}

Biomarker assessment of gastroenteropancreatic neuroendocrine tumor (GEP-NET) disease has been difficult since the default is provided by a monoanalyte measurement, chromogranin A, which has well-described limitations (Lawrence et al. 2011, Marotta et al. 2012). The current scientific paradigm for biomarker development has focused on the advancement of multianalyte technologies (Engels et al. 2013, Grimm et al. 2013). 
This strategy facilitates the coupling of integral aspects of disease represented by individual markers into a mathematical algorithm that provides multidimensional clinical and pathobiological information inaccessible in a monoanalyte approach (Modlin et al. 2014a,b).

In seeking to advance beyond a monoanalyte strategy, we developed a genetic biomarker assay in blood for GEPNETs based upon qRT-PCR measurement of 51 circulating NET marker genes. Normalized gene expression is classified using four different learning algorithms (support vector machine, linear discrimination analysis, K-Nearest Neighbor, and Naive Bayes (Bayes)) and a score is assigned based on a majority vote (Modlin et al. 2013a). Output is expressed as a $0-8$ score, where a score $>2$ is classified as a GEP-NET (Modlin et al. 2014a,b). The PCR test (NET score) is standardized and reproducible (inter- and intra-assay coefficient of variation (CV) $<2 \%$ ), and not affected by age, gender, ethnicity, fasting, or PPI medication (Modlin et al. 2014c). The score is robust (inter- and intra-assay CV $<2 \%$; Modlin et al. 2014c) with a high level of sensitivity and specificity (98 and 97\% respectively). This multianalyte algorithmic assay (MAAA) strategy is significantly more accurate as a circulating biomarker than other currently utilized monoanalytes (Z-statistic 4.85-5.9, $P<0.0001$ vs e.g. chromogranin A and pancreastatin) for NET detection (Modlin et al. 2013a, 2014a,b,c,d).

Our current goal was to advance from a linear interpretation of disease activity based upon quantification of gene expression ( $0-8$ score) to an amplified algorithm that incorporated gene clusters (GC) representative of NET neoplasia. This cluster integrated algorithm (CIA) would thus comprise both the diagnostic blood transcripts and include the mathematical expression levels of specific GC that delineate a variety of neoplastic biological processes, elegiacally referred to as the 'hallmarks of cancer' (Hanahan \& Weinberg 2000, 2011).

The 51 marker gene score includes a series of genes that are associated with neoplastic behavior. Captured by gene co-expression networks from tissue and blood transcriptome databases (Modlin et al. 2013a, Kidd et al. 2014), these include biologically relevant transcripts involved in NET proliferation, signaling and secretion (Wulbrand et al. 1998, Kidd et al. 2006, Karhoff et al. 2007, Drozdov et al. 2009, Cui et al. 2010, Muscarella et al. 2011) as well as genes reported to have a defined association with tumor initiation and metastasis (Hogarty et al. 2008, Kim et al. 2008, Miretti et al. 2008, Naik et al. 2009, Bralten et al. 2010). As such, they overall provide 'omic' information.

The NET score provides a robust circulating 'fingerprint' for GEP-NET detection using a linear score (0-8).
It has not, however, been defined whether measurements of circulating transcripts functions as a multianalyte biomarker that correlates with tumor tissue expression levels. We postulated that if this was demonstrable, then the blood derived NET score could be used to define the real-time biological and clinically relevant state of a tumor. Thus, repetitive 'liquid biopsies' (blood sampling) would provide direct information about the tumor, its pathophysiology and its response to intervention. Therefore, a specific aim of the investigation was to define whether blood transcript levels directly correlated with tumor tissue levels collected at the same time point (i.e. at surgery).

Hanahan \& Weinberg $(2000,2011)$ have elegantly derived a synopsis of the complexity of cancer pathobiology by delineating a sequence of 'hallmarks' pathognomonic of neoplasia. These descriptors have been confirmed using systems biology approaches (Wang et al. 2014) and 'cancer hallmark networks' can be quantified and computationally modeled to predict cancer evolutionary paths as well as clinical phenotypes. We utilized our pipeline to develop a systems biology approach to identify candidate NET biomarker genes. We hypothesized such genes would be 'neoplasia-relevant' and could be categorized using a 'hallmark' approach as a template. A mathematical algorithm (NETest) could then be derived that would capture the 'biology' of NET neoplasia. By inference, we would predict that the identification of such hallmarks would allow for extrapolation to provide clinically useful information. The corollary is that effective treatment protocols could be defined by their ability to modify transcript clusters or conversely ineffective agents would fail to perturb the cluster signatures. To this end, we examined whether the 51 marker gene panel could be mathematically reconfigured to include specific neoplastic defining 'hallmarks' or 'omes'. Our concept was to evaluate NET selectivity, determine whether tissue levels of these hallmarks were increased compared to non-neoplastic tissue and confirm that these GC could be not only measured in circulating blood samples but that they correlated with tissue expression levels. Finally, we sought to identify whether hallmark expression differed between two broad clinical groups currently characterized in clinical vernacular as stable disease (SD) or progressive disease (PD). Identification of biologically relevant 'omes' enabled us to expand on the original algorithm to include biologicallyrelevant gene expression data.

\section{Methods}

Publically available NET microarrays ( $n=2$ sets: described below; Kidd et al. 2014) were assessed (group 1). These

Published by Bioscientifica Ltd 
included sample set 1 which comprised nine NET transcriptomes (obtained from the small intestine) and normal small intestinal mucosa (Affymetrix U133A chips, $n=9$ tumors and $n=3$ normal mucosa, ArrayExpress: E-GEOD-6272) (Kidd et al. 2006) and sample set 2 which comprised six NET transcriptomes (Affymetrix U133 Plus2 chips, $n=3$ primary midgut NETs and $n=6$ normal mucosa, ArrayExpress: E-TABM-389) (Leja et al. 2009).

Other samples (groups 2 and 3) were collected and analyzed according to a standard IRB protocol (Yale University, 6/17/2013) in accordance with the World Medical Association Declaration of Helsinki (Modlin et al. 2013a). Group 2 included 22 pathologically verified samples that were provided by Yale University (Surgical Pathology Tissue Databank per standard IRB protocol: Yale University, $6 / 17 / 2014)$ as well as normal mucosa $(n=8)$. The grade stage as well as previous treatment are included in Table 1.
Matched blood samples were also available in nine. Group 3 included blood samples included in a training set ( $n=130$ (previously described); Modlin et al. 2013a) as well as samples from an independent set $(n=159$ : clinically stable disease (SD) $n=111 ; \mathrm{PD} n=48$ ). The demographics of the 63 NETs in the training set were mean age 56 years (range: $18-80$ ) and gender distribution (M:F) of 33:30. The 159 NETs in the independent set were mean age 57.1 years (range: 27-83), gender distribution (M:F) of 86:73. Tumor site, grade, stage, and treatment are included in Table 2 . The sets were well-matched, except the independent set had more pancreatic NETs $\left(\chi^{2}=30\right.$, $P<0.00001)$. Each group included both 'SD' and 'PD'. SD was assessed as per standard clinical criteria and RECIST criteria. The PD group (disease progression) was based on review of changes in radiological and nuclear medicine images at tumor board using the same criteria. PD was

Table 1 Demographics of NETs (sample sets one to three; tissue samples)

\begin{tabular}{|c|c|c|}
\hline Sample set & Sample no. & Gender \\
\hline Normal & N1 & $\mathrm{M}$ \\
\hline Normal & N2 & $\mathrm{F}$ \\
\hline Normal & N3 & $\mathrm{F}$ \\
\hline Normal & N4 & $\mathrm{M}$ \\
\hline Normal & N5 & $F$ \\
\hline Normal & N6 & $\mathrm{M}$ \\
\hline Normal & N7 & $\mathrm{F}$ \\
\hline Normal & N8 & $\mathrm{M}$ \\
\hline Tumor & T1 & $\mathrm{M}$ \\
\hline Tumor & $\mathrm{T} 2$ & $\mathrm{M}$ \\
\hline Tumor & T3 & $\mathrm{F}$ \\
\hline Tumor & T4 & $\mathrm{M}$ \\
\hline Tumor & T5 & $\mathrm{F}$ \\
\hline Tumor & T6 & $\mathrm{F}$ \\
\hline Tumor & $\mathrm{T7}$ & $F$ \\
\hline Tumor & T8 & $\mathrm{F}$ \\
\hline Tumor & T9 & $\mathrm{M}$ \\
\hline Tumor & T10 & $\mathrm{M}$ \\
\hline Tumor & T11 & $F$ \\
\hline Tumor & $\mathrm{T} 12$ & $\mathrm{M}$ \\
\hline Tumor & T13 & $\mathrm{F}$ \\
\hline Tumor & T14 & $\mathrm{F}$ \\
\hline Tumor & T15 & $\mathrm{M}$ \\
\hline Tumor & T16 & $\mathrm{M}$ \\
\hline Tumor & $\mathrm{T} 17$ & $\mathrm{M}$ \\
\hline Tumor & T18 & $F$ \\
\hline Tumor & T19 & $\mathrm{F}$ \\
\hline Tumor & T20 & $\mathrm{M}$ \\
\hline Tumor & T21 & $\mathrm{M}$ \\
\hline Tumor & T22 & $\mathrm{M}$ \\
\hline
\end{tabular}

\begin{tabular}{|c|c|}
\hline Age & Site \\
\hline 46 & Small intestine \\
\hline 62 & Small intestine \\
\hline 48 & Colond \\
\hline 65 & Colon $^{d}$ \\
\hline 89 & Colon $^{d}$ \\
\hline 41 & Colon $^{d}$ \\
\hline 66 & Rectum $^{d}$ \\
\hline 66 & Rectum $^{d}$ \\
\hline 70 & Stomach $^{\mathrm{e}}$ \\
\hline 80 & Pancreas \\
\hline 60 & Pancreas \\
\hline 52 & Pancreas \\
\hline 64 & Pancreas ${ }^{\mathrm{e}}$ \\
\hline 75 & Small Intestine \\
\hline 68 & Small Intestine \\
\hline 61 & Small Intestine \\
\hline 67 & Small Intestine \\
\hline 69 & Small Intestine \\
\hline 62 & Small Intestine \\
\hline 75 & Small Intestine \\
\hline 61 & Small Intestine \\
\hline 59 & Small Intestine \\
\hline 42 & Small Intestine \\
\hline 45 & Appendix ${ }^{\mathrm{e}}$ \\
\hline 50 & Rectum \\
\hline 47 & Rectum \\
\hline 51 & Rectum \\
\hline 70 & Rectum \\
\hline 48 & Rectum ${ }^{\mathrm{e}}$ \\
\hline 66 & Rectum \\
\hline
\end{tabular}

\begin{tabular}{l}
\multicolumn{1}{c}{ Source } \\
\hline Normal tissue \\
Normal tissue \\
Normal tissue \\
Normal tissue \\
Normal tissue \\
Normal tissue \\
Normal tissue \\
Normal tissue \\
Primary \\
Primary \\
Primary \\
Metastasis \\
Metastasis \\
Metastasis \\
Primary \\
Metastasis \\
Metastasis \\
Metastasis \\
Primary \\
Primary \\
Primary \\
Primary \\
Metastasis \\
Primary \\
Primary \\
Primary \\
Metastasis \\
Metastasis \\
Metastasis \\
Primary
\end{tabular}

\begin{tabular}{c} 
Grade $^{\mathrm{a}}$ \\
\hline NA \\
NA \\
NA \\
NA \\
NA \\
NA \\
NA \\
NA \\
2 \\
1 \\
1 \\
2 \\
2 \\
2 \\
1 \\
2 \\
1 \\
1 \\
1 \\
1 \\
1 \\
1 \\
1 \\
1 \\
1 \\
1 \\
1 \\
1 \\
2 \\
1 \\
\hline
\end{tabular}

\begin{tabular}{c}
\hline Stage $^{\text {b }}$ \\
\hline NA \\
NA \\
NA \\
NA \\
NA \\
NA \\
NA \\
NA \\
II \\
II \\
I \\
IV \\
IV \\
IV \\
II \\
IV \\
IV \\
IV \\
II \\
II \\
II \\
II \\
IV \\
I \\
II \\
I \\
IV \\
IV \\
IV \\
I
\end{tabular}

\begin{tabular}{l}
\hline Treatment \\
\hline Surgery - CD \\
Surgery - CD \\
Surgery - UC \\
Surgery - CCa \\
Surgery - CCa \\
Surgery - CCa \\
Surgery - RCa \\
Surgery - RCa \\
Surgery (treatment naïve) \\
Surgery (treatment naïve) \\
Surgery (treatment naïve) \\
Surgery (prior IFN, chemo) \\
Surgery (prior chemo) \\
Surgery (prior chemo) \\
Surgery (treatment naïve) \\
Surgery (treatment naïve) \\
Surgery (prior SSA) \\
Surgery (prior SSA) \\
Surgery (treatment naïve) \\
Surgery (treatment naïve) \\
Surgery (treatment naïve) \\
Surgery (treatment naïve) \\
Surgery (treatment naïve) \\
Surgery (treatment naïve) \\
Surgery (treatment naïve) \\
Surgery (treatment naïve) \\
Surgery (treatment naïve) \\
Surgery (treatment naïve) \\
Surgery (prior SSA) \\
Surgery (treatment naïve) \\
\end{tabular}

F, female; M, male; N, no; Y, yes; CCa, colon cancer; CD, Crohn's disease; RCa, rectal cancer; UC, ulcerative colitis; NA, not applicable.

${ }^{a} G$ raded per ENETs criteria (Rindi et al. 2006, 2007).

${ }^{b}$ Staged per ENETs criteria (Rindi et al. 2006, 2007).

'Treatment included somatostatin analogs (SSA), interferon (IFN), or chemotherapy (chemo).

${ }^{\mathrm{d}}$ Microscopically and macroscopically normal mucosa. Samples were collected at surgery for the indicated diseases. All tissue was pathologically verified as 'normal'.

Included matched blood samples. 
Table 2 Clinical characteristics of patients (test and independent validation sets; blood samples)

\begin{tabular}{lcc}
\hline & \multicolumn{2}{c}{ Study set } \\
\cline { 2 - 3 } & & \multicolumn{2}{c}{$\begin{array}{c}\text { Independent set } \\
(n=159)^{\mathrm{a}, \mathrm{c}}\end{array}$} \\
\cline { 3 - 4 } $\begin{array}{c}\text { Primary location } \\
\text { Lung }\end{array}$ & $3(5 \%)$ & $5(3 \%)^{\mathrm{d}}$ \\
Stomach & $4(6 \%)$ & $6(4 \%)^{\mathrm{d}}$ \\
Pancreas & $3(5 \%)$ & $43(27 \%)^{\mathrm{d}}$ \\
SI & $39(62 \%)$ & $82(52 \%)^{\mathrm{d}}$ \\
Appendix & $8(13 \%)$ & $3(2 \%)^{\mathrm{d}}$ \\
Colorectal & $6(9 \%)$ & $7(4 \%)^{\mathrm{d}}$ \\
CUP & $0(0 \%)$ & $12(8 \%)^{\mathrm{d}}$ \\
Grade & & \\
G1 & $30(48 \%)$ & $87(55 \%)$ \\
G2 & $18(28 \%)$ & $29(18 \%)$ \\
G3 & $2(3 \%)$ & $5(3 \%)$ \\
ND & $13(21 \%)$ & $38(24 \%)$ \\
Stage & & \\
I & & $11(7 \%)$ \\
II & $7(11 \%)$ & $16(10 \%)$ \\
III & $5(8 \%)$ & $0(0 \%)$ \\
IV & $0(0 \%)$ & $121(76 \%)$ \\
ND & $46(73 \%)$ & $11(7 \%)$ \\
\hline
\end{tabular}

CUP, carcinoid of unknown primary; ND, no data available; SI, small intestine. The grade and stage distribution was not significantly different between the stable and progressive disease groups. The majority $>95 \%$ of patients were Caucasian.

${ }^{a}$ Comparison of the two sets with the spectrum of disease included in the Surveillance Epidemiology and End Results (SEER) database (GEP-NETs) identified no significant differences indicating that the patient characteristics provided a reasonable reflection of the clinical spectrum of NET disease.

${ }^{\mathrm{b}}$ This included stable disease $(n=35)$ and progressive disease $(n=27)$.

'This included stable disease $(n=111)$ and progressive disease $(n=48)$.

dPrimary location distribution was significantly different in the two sets $\left(\chi^{2}=30.02, P<0.0001\right)$. Both the grade and stage were similar $\left(\chi^{2}=1.3-2.9\right.$, $P=0.4-0.7)$.

${ }^{\text {e}}$ GEP-NETs grade based on Ki67 or mitotic index (ENETs guidelines (Rindi et al. 2006, 2007)). BP grade based on WHO guidelines (Travis et al. 2004). 'Stage based on ENETs guidelines (Rindi et al. 2006, 2007). Tumor disease was identified in lymph nodes, mesentery, liver, lung, bone, and ovary (or any combination thereof). Methodologies including octreoscan, computed tomography $(\mathrm{CT})$, magnetic resonance imaging (MRI), identification at surgery, and identification at pathology, e.g. positive lymph nodes.

defined as any increase in tumor burden (any individual lesion), while SD was defined as no increase in tumor burden. The grade and stage distribution was not significantly different.

All individuals who provided blood were (6/20093/2014) from the Yale School of Medicine, Smilow Cancer Center outpatient clinics and provided informed consent. Blood samples $\left(5 \mathrm{ml}\right.$ ) were collected in $9 \mathrm{mg} \mathrm{K} \mathrm{K}_{2}$ EDTA tubes (BD Vacutainer Venous Blood Collection Tubes, BD Diagnostics, Franklin, NJ, USA). Aliquots of whole blood were stored at $-80{ }^{\circ} \mathrm{C}$ within $2 \mathrm{~h}$ of collection (samples immediately stored on ice $/ 4^{\circ} \mathrm{C}$ after sampling) per standard molecular diagnostics protocols for PCR-based studies (Raza et al. 2012).

\section{Group 1: transcriptome assessment}

Gene expression (fold change) of each of the target genes was examined in two publically available datasets, sample set 1 (Kidd et al. 2006) and sample set 2 (Leja et al. 2009). Individual analyses were performed using the web-based GeneProfiler tool (GeneProfiler, Bering Limited, http:// beringresearch.com/geneprofiler). Primary tumors were compared with non-matched normal mucosal samples. Sample set 1 consisted of 22283 probes and 12 arrays, while sample set 2 consisted of 54675 probes and 12 arrays. Probe sets that were unlikely to be reliable were eliminated using detection of present/absent calls. Probes present in $>50 \%$ of samples were retained (McClintick \& Edenberg 2006). Raw probe intensities were normalized using the Robust Microarray Average approach (Irizarry et al. 2003). Array outlier detection was performed in the array Quality Metrics package (Kauffmann et al. 2009) using the Kolmogorov-Smirnov statistic between each array's distribution and the distribution of the pooled data. To enhance microarray annotation, probe identifiers (IDs) were mapped to Entrez Gene IDs (accessed 7th April 2013; Maglott et al. 2011). In cases where multiple probes mapped to the same Entrez ID, the average probe intensity was calculated. Probes without an Entrez record were removed from analysis. Genes that were consistently identified as differentially expressed using multiple ranking algorithms (Boulesteix \& Slawski 2009; fold change ranking, ordinary $t$-statistic, shrinkage $t$-statistic, limma, significance analysis of microarrays) were called significant and retained for further analysis. This approach ensured that differential expression analysis was i) unbiased and ii) consistent across different array platforms.

\section{Tissue RNA isolation and real-time PCR}

RNA was extracted (TRIzol, Invitrogen; Kidd etal. 2005, 2014) and real time RT-PCR analysis was performed using Assayson-Demand products and the ABI 7900 Sequence Detection System according to the manufacturer's suggestions (Kidd et al. 2005, 2014). Cycling was performed under standard conditions (TaqMan Universal PCR Master Mix Protocol) and data normalized (using $A L G 9$ and the $\Delta \Delta C_{\mathrm{T}}$ method (Microsoft Excel)) using small bowel mucosa as a control. Samples were evaluated and scored using the MATLABderived protocol for blood (Modlin et al. 2013a,b, 2014c). As a comparison normal tissue samples $(n=8)$ were included.

\section{Blood-based MAAA PCR test}

We used a two-step manual technique protocol (RNA isolation with cDNA production and qPCR). Transcripts

Published by Bioscientifica Ltd. 
(mRNA) were isolated from $1 \mathrm{ml}$ EDTA-collected blood samples using the mini blood kit (Qiagen). The RNA quantity was $50 \mu \mathrm{l}$, the quality was $>1.8\left(\mathrm{~A}_{260: 280}\right.$ ratio); analysis of the RNA pattern on electrophoresis (Agilent Technologies, Santa Clara, CA, USA) RNA integrity number (RIN) > 5.0 (Fleige et al. 2006). The standard Qiagen isolation protocol (heme/gDNA contamination not detected) with no modifications was used. cDNA was produced from $50 \mu \mathrm{l}$ RA using a High Capacity Reverse Transcriptase Kit (Life Technologies: cDNA production 2000-2500 ng/ $\mathrm{\mu l}$ ) and stored at $-80^{\circ} \mathrm{C}$. QPCR was performed (384-well plate, HT-7900) with the cDNA $(200 \mathrm{ng} / \mu \mathrm{l})$ and $16 \mu \mathrm{l}$ of reagents/ well (Universal Master Mix II with UNG, Life Technologies, triplicate wells) $\left(50{ }^{\circ} \mathrm{C} 2 \mathrm{~min}, 95^{\circ} \mathrm{C} 10 \mathrm{~min}\right.$, then $95^{\circ} \mathrm{C} 15 \mathrm{~s}$, $60{ }^{\circ} \mathrm{C}, 60 \mathrm{~s}$ for 40 cycles) as described (Modlin et al. 2013a, 2014c). A NET score (0-8) was derived from the PCR data using MATLAB (R2011a, Mathworks, Natick, MA, USA; Modlin et al. 2013b); a value $\geq 2$ is a positive tumor score (Modlin et al. 2013a,b, 2014c). Quantification of gene expression in the clusters ('omes') was undertaken by summation of individual gene expression. For example, the 'SSTRome' comprises the summated gene expression of SSTR1, SSTR3, and SSTR5. Individual 'omes' that were significantly different $(P \leq 0.002)$ between SD and PD and were elevated in GEP-NET blood compared to control blood were included in an activity algorithm. Six 'omes' (SSTRome, proliferome, metabolome, secretome (II), epigenome, and plurome) met this criterion and were summated. Nonsignificant and therefore excluded 'omes' included secretome (I), signalome, and apoptome. A cut-off of 80 (summated normalized gene expression of six 'omes') based on receiver operating characteristic (ROC) analyses was used to differentiate disease phenotypes (SD vs PD). The original 0-8 score derived from the blood-based gene test that was used for the diagnosis of NETs was then reconfigured using the activity algorithm to generate a CIA. The latter was utilized to generate a disease activity range expressed as logarithmic basis of activity ranging from 0 to $100 \%$. This was generated by weighting the original score by whether it was 'stable' or 'progressive'. Scores of 0-8 with 'stable' phenotype were converted to 0-44\% (low activity); those with 'progressive' phenotype ranged from 44 to $100 \%$ (high activity).

\section{Statistical analysis}

Sensitivity comparisons using $\chi^{2}$, non-parametric measurements and ROC analysis were made between the standard MAAA-PCR and NETest. Both Prism 6.0 for Windows (GraphPad Software, La Jolla, CA, USA, www.graphpad.com) and MedCalc Statistical Software version 12.7.7 (MedCalc Software bvba, Ostend, Belgium; http://www.medcalc.org) were utilized. The accuracy of each of the MAAAs was compared using ROC curve analyses (continuous variables). The sensitivity, specificity, and the area under the curve (AUC) were calculated (MedCalc Software bvba) (Hanley \& McNeil 1982) for AUC comparison and derivation of the $Z$-statistic (Hanley \& McNeil 1983) (MedCalc Software bvba).

\section{Results}

\section{Assessment of circulating NETest genes expression in NET tumor tissue}

To confirm the 51 marker gene blood panel captured NET genes, we examined gene expression in two publically available datasets (Kidd et al. 2007, Leja et al. 2009). All 51 genes were identified. Forty-five (92\%) were elevated in NETs compared to normal mucosa at a transcriptome level (Fig. 1A). A second group of pathologically verified GEP-NET samples $(n=22)$ examined by qRT-PCR identified 50 of 51 genes $(98 \%)$ to be elevated in NETs compared to normal mucosa (Fig. 1B). Examination of normal tissue $(n=8)$ demonstrated all to score between 0 and 2, thus none were classified as NETs (Modlin et al. 2013a, 2014a,b; Table 3). Twenty-two (100\%) of the pathologically verified tumor samples exhibited scores $>2$ and were classified as NETs per protocol (Modlin et al. 2013a, 2014a,b; Table 3). A subanalysis of different organ sites identified that the NET score accurately (100\%) classified samples from the stomach $(1 / 1$ : activity: $80 \%)$, pancreas (4/4: $55 \pm 3 \%)$, small intestine (10/10: $52 \pm 6 \%)$, appendix (1/1: $47 \%)$, and rectum (6/6: $58 \pm 3 \%)$.

\section{Correlation between circulating and tissue NETest genes}

Pre-surgical blood samples were available for nine of the 22 pathologically verified tumor tissue samples (staging included: I $(n=1)$, II $(n=3)$, and IV $(n=5)$. Seven control blood (non-NET) and matched normal mucosa were also available. Comparison analysis identified that the blood NET scores closely correlated (two-tailed non-parametric correlation analysis Spearman), $r=0.804, P=0.0004$ with tumor tissue samples. In addition, the accuracy, i.e. the ability of the scores to classify a sample as either 'normal' or 'tumor' for matched tissue and blood samples, was 100\% (Fig. 2A). An assessment of each of the 51 marker genes individually identified significant expression correlation in tumor tissue and blood respectively. Representative samples from stomach, pancreas, small intestine and

Published by Bioscientifica Ltd 

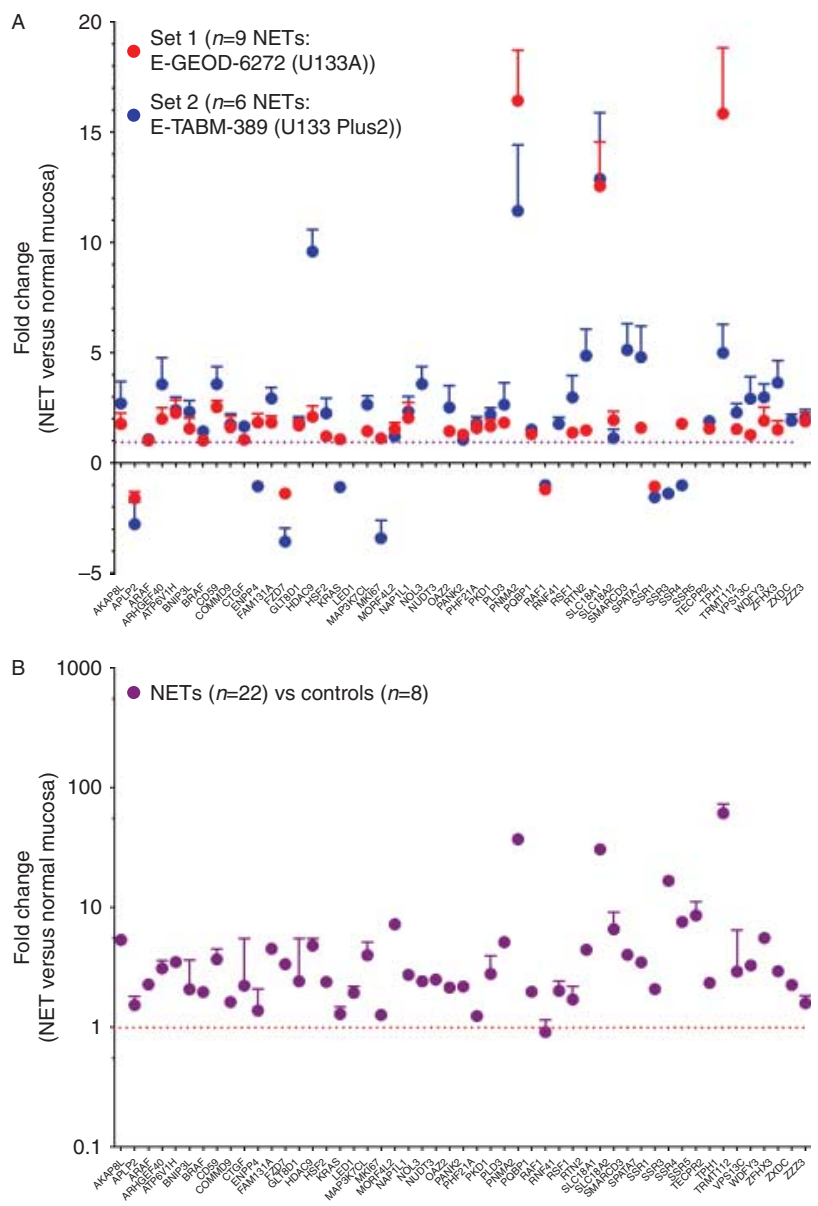

\section{Figure 1}

Marker gene expression in tissues. (A) Fold changes (FC) of differentially expressed genes in neuroendocrine tumors compared to normal mucosa for each of the two sample transcriptome sets. Differentially expressed genes have a FC $>1$. Forty-seven of the 51 genes were upregulated at a transcriptome level $(P<0.05)$. (B) FC of differentially expressed genes in NETs $(n=22)$ compared to normal mucosa $(n=8)$. Differentially expressed genes have a FC $>1$. Fifty of the 51 genes were determined to be upregulated at a transcriptional level. Mean \pm s.E.M.

rectum are included (linear regression analysis (Pearson), goodness of fit $R^{2}=0.09-0.26, P=0.002-0.04$; Fig. 2B).

\section{Derivation and tissue expression of the NET 'hallmarks'}

Thirty of the 51 genes (58\%) could be classified into nine 'hallmarks' or GC (Table 4). Clusters were based on a combination of classical descriptors (Hanahan \& Weinberg 2000, 2011) as well as the protein: protein interactome network (Franceschini et al. 2013, Kidd et al. 2014). A heat map analysis of each of the clusters or 'omes' identified expression was higher in NETs than in normal mucosa (Fig. 3A). Expression in 22 NETs when compared to normal mucosa $(n=8)$ identified that seven of the clusters were specific to NETs (Fig. 3B, ${ }^{*} P<0.01$, ${ }^{* *} P<0.05$ Mann-Whitney $U$ test, two tailed). Fold change analysis (Mann-Whitney $U$ test, two tailed) identified significant increases in seven clusters. One cluster, the 'plurome', was significantly reduced in tumor tissue compared to normal mucosa, while the proliferome was similar between NETs and normal tissue (Fig. 3C).

\section{Correlation of NET 'Hallmarks' between tumor tissue and blood}

We next examined 'omes' in the nine matched tumor and blood samples to examine correlation. No significant differences were noted between clusters in tissue and blood ( $P=0.11$, Wilcoxon matched-pairs signed rank test, $r_{\mathrm{s}}$ (Spearman effectiveness of pairing): $0.41, P=0.0026$ (one-tailed)). Individual 'omes' were higher in blood than tissue except for the secretome (Fig. 4A). No significant differences were noted between each of the individual 'omes' in blood and tissue ( $P=0.1-0.9$; Wilcoxon matchedpairs signed rank test).

\section{Clinical utility of clustered gene expression}

Thereafter, we assessed the expression of each of the 'omes' in peripheral blood samples to examine whether expression levels were related to clinically defined SD or PD as determined by best clinical judgment and/or imaging data. In the test set (initially used to develop the NET score (Modlin et al. 2013a; $n=130$ : controls: $n=67$, NETs: $n=63$ (SD: $n=35$ and PD: $n=28)$ ), significant differences were noted in 'omes'. Non-parametric testing (Kruskal-Wallis) identified that eight of the 'omes' differed $(P=0.01-$ $P<0.0001$, Kruskal-Wallis statistic: 9.1-51.7 (Table 5). The metabolome was not identified to be different in this analysis $(P=0.43)$. Multiple testing with adjustments (Dunn's multiple testing comparison) identified where SD or PD were significantly different and when they differed to controls. Data are included in Fig. 4B. These data demonstrate that GC not only differentiate SD and PD from controls but segregate the two clinical groups of SD and PD.

Table 3 NETest scores in tissue samples

\begin{tabular}{|c|c|c|c|}
\hline & Score (0-8) & Classification & $\begin{array}{c}\text { Concordance with } \\
\text { histopathology } \\
(\%)\end{array}$ \\
\hline $\begin{array}{l}\text { Normal mucosa } \\
\quad(n=8)\end{array}$ & $0.625 \pm 0.26$ & $8 / 8$ 'normal' & 100 \\
\hline NETs $(n=22)$ & $4.42 \pm 0.23$ & $22 / 22$ 'tumor' & 100 \\
\hline
\end{tabular}

Published by Bioscientifica Ltd. 

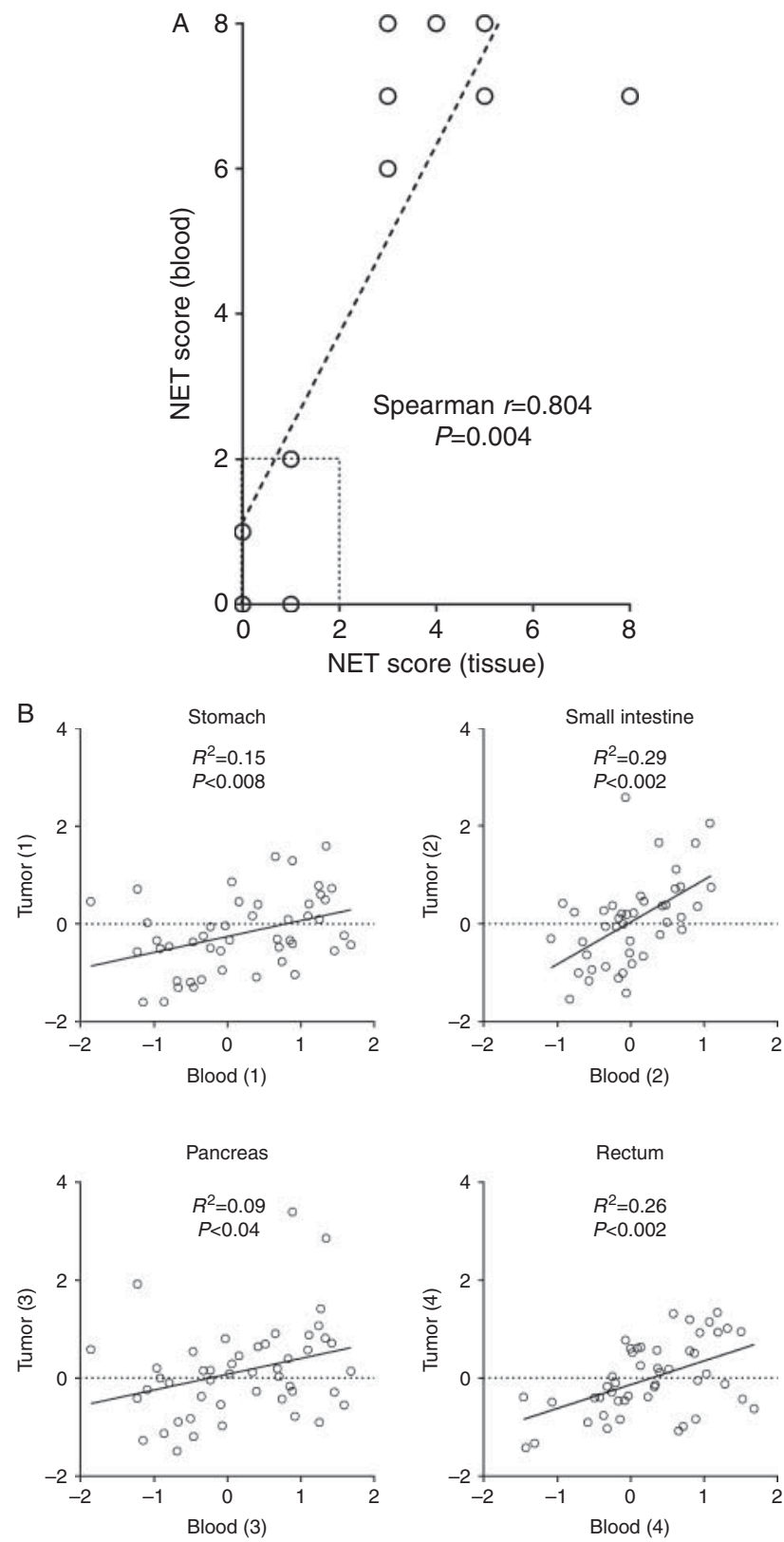

Figure 2

Correlation between marker gene expression in tissue and in peripheral blood. (A) Linear regression analysis of NET scores from matched tissue and blood samples demonstrating the scores (scale 0-8) were highly correlated (Spearman $r=0.804, P=0.0004$ (two-tailed)). All normal samples had NET scores $<2$, all matched tumors had NET scores $>2$. (B) Correlation between each of the 51 marker genes in matched tumor tissue and circulating blood. Gene expression was log transformed. $R^{2}$ values (linear regression, Pearson: goodness of fit) ranged from 0.09 (pancreatic, $P<0.04$ ) to 0.29 (small intestine, $P<0.002$ ).

We next examined cluster gene results in the independent set ( $n=159$ : including SD: $n=111$ and PD: $n=48$ ), evaluating each of the clusters in SD vs PD. In this set, the SSTRome, proliferome, secretome (II), plurome, and

epigenome were significantly increased $(P<0.05$, MannWhitney $U$ test) in PD compared to SD (Fig. 4C). Receiver operator curve analysis of the combined dataset (test and independent sets) identified that the AUC for differentiating SD $(n=146)$ from PD $(n=75)$ was significant for seven of the omes (AUC: $0.65-0.72, P<0.0002$, Table 5). This was not significant for the growth factor signalome or the general secretome. These data confirm that GC expression can be used to differentiate between SD and PD.

\section{Cluster analysis integration into the blood multianalyte algorithm}

We examined the NET scores (0-8) of the complete patient group (test set+independent set: $n=222$; excluding controls) in SD vs PD. In the SD group $(n=146)$, the highest frequency score was $4(30 \%$, Fig. 5A), while $46 \%$ of PD group $(n=75)$ had a score of 8 (Fig. $5 \mathrm{~A})$. A risk probability assessment identified that NET scores ranging from 0 to 5 were associated with SD with a $\geq 90 \%$ certainty (Fig. 5B). A score of 8 was considered as PD (>90\%). However, scores of 6 and 7 could not accurately differentiate SD vs PD. We next examined whether individual 'omes' could differentiate between SD and PD. ROC analyses identified that the AUC's ranged from 0.514 (secretome I) to 0.72 (plurome) (Table 4 and Fig. 5C).

The omes $(n=6)$ that were significantly increased $(P \leq 0.002)$ in PD compared to SD were then included in an activity algorithm (omes were summated into a single score). The 'apoptome' was excluded since it was decreased compared to control blood (Fig. 4B). The resultant activity algorithm was tested in the combined set and noted to have an AUC of $0.81 \pm 0.03$ (95\% CI: 0.753-0.861), $P<0.0001$ (Fig. 5D) for differentiating between PD and SD.

The clinical utility of this algorithmic reconfiguration was then assessed by re-evaluating the original MAAA derived $0-8$ score. We weighted the 0 to 8 score by whether the sample was called SD or PD by the algorithm.

Table 4 Hallmarks of NETs

Proliferome

Growth factor signalome

Metabolome

Secretome I (general)

Secretome II (progressive)

Epigenome

Apoptome

Plurome

SSTRome
Ki67, NAP1L1, NOL3, TECPR2

ARAF1, BRAF, KRAS, RAF1

ATP6V1H, OAZ2, PANK2, PLD3

PNMA2, VMAT2

PQB1, TPH1

MORF4L2, NAP1L1, PQB1, RNF41, RSF1, SMARCD3, ZFHX3 BNIP3L, WDFY3

COMMD9

SSTR1, SSTR3, SSTR4, SSTR5 
A

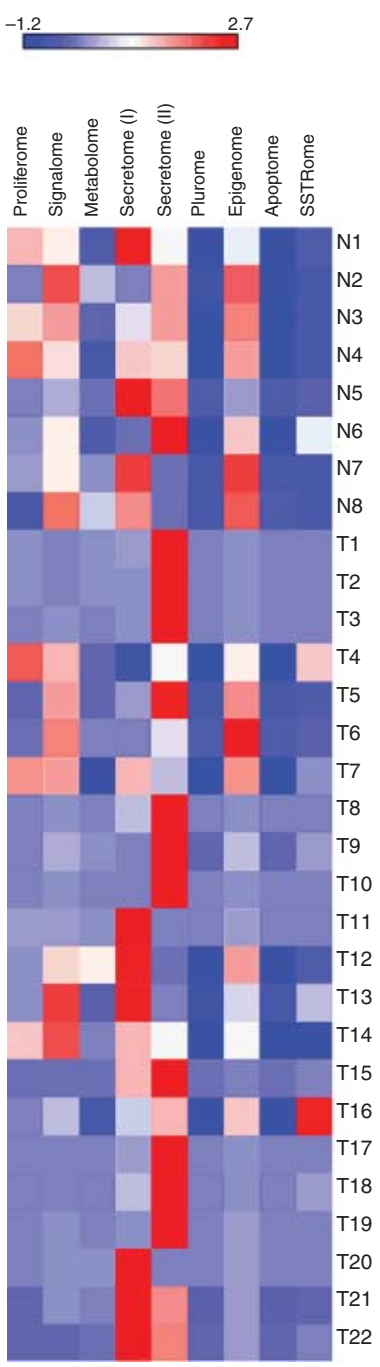

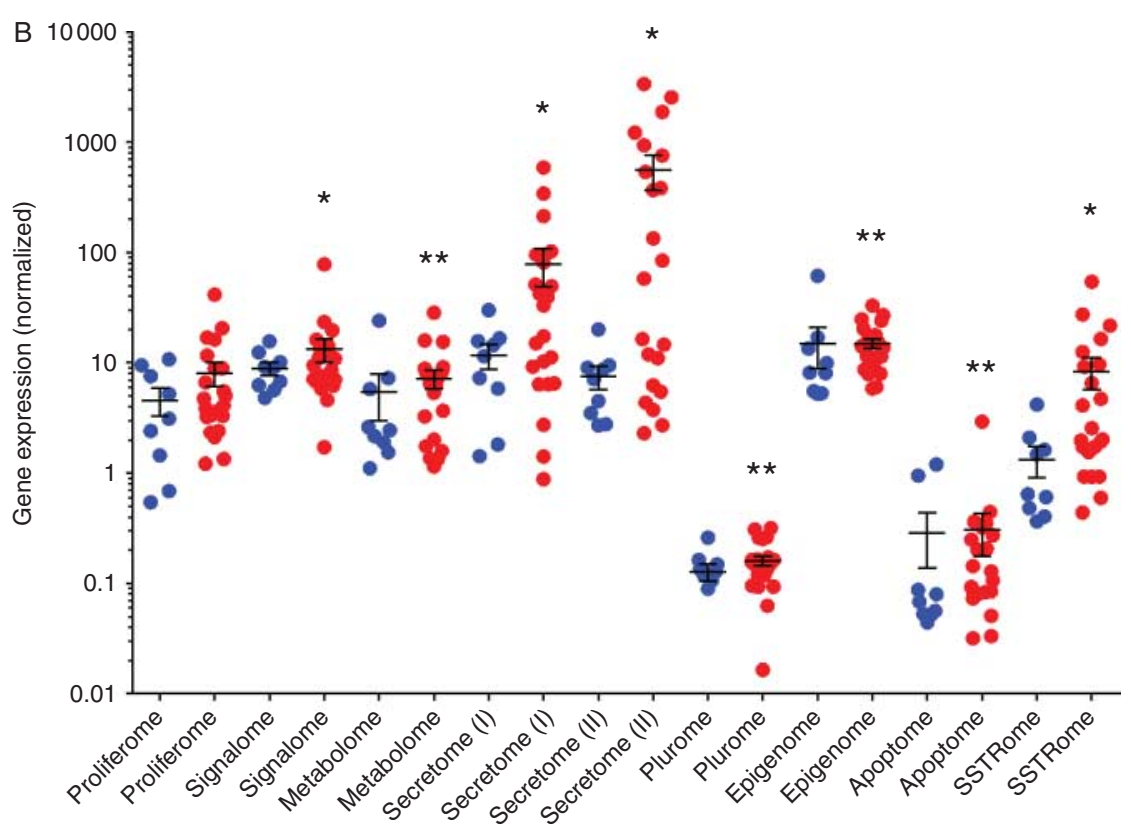

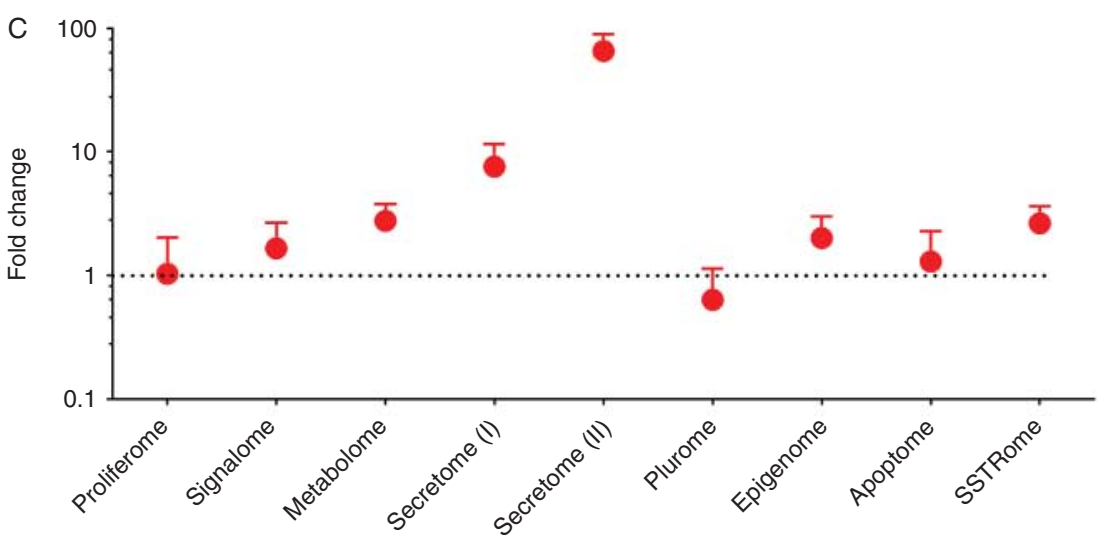

Figure 3

Gene expression classified by 'ome' in neuroendocrine tumors and normal mucosa. (A) Heatmap of 'omes' in normal (N1-N8) mucosa and in NETs (T1-T22). Each of the columns are normalized to a mean of 0 and a variance of 1. Expression levels are plotted between -2 and 5 . NETs express higher levels of the omes. (B) Cluster assessment in normal mucosa (blue) and NETs (red). Expression of the signalome, metabolome, secretome (I) and (II), epigenome, apoptome and SSTRome were significantly elevated

This allowed the development of a $0-100 \%$ activity score that incorporated both the machine-learning derived 0-8 score with the additional information regarding disease activity (as captured in the 'ome' algorithm; Fig. 6A). The distribution of activity scores is included in Fig. 6B. PD samples had significantly higher activity scores than SD samples $(83.7 \pm 24.4 \%$ vs $34.1 \pm 27 \%, P<0.0001)$. The upper $95 \%$ CI for SD was $45 \%$ indicating this could be used as a cut-off to differentiate PD from SD. Using this value
(Mann-Whitney $U$ test) in NETs. Genes in the plurome were decreased in NETs. Values are included in individual scatter dot plots for each 'ome' (data is presented logarithmically, mean \pm S.E.M.). ${ }^{*} P<0.01, * * P<0.05$ vs normal mucosa. (C) Fold changes (FC) of the 'omes' in NETs $(n=22)$ compared to normal mucosa $(n=8)$. Differentially expressed genes have a FC $>1$. All 'omes' except for the plurome were $>1$. Data is presented logarithmically, mean \pm s.D.

(45\%), the accuracy was $87.4 \%$ for predicting disease status as stable or progressive. The metrics for this, undertaken in the same set $(n=222)$, were sensitivity: $91.1 \%$, specificity: $85.3 \%$, positive predictive value: $77.4 \%$, and negative predictive value: $94.6 \%$ (Fig. 6C). ROC analysis demonstrated an AUC of $0.92 \pm 0.02$ (95\%CI: 0.88-0.96), $P<0.0001$. A comparison between the CIA NETest (0-100\%) and the NET score (0-8) demonstrated the former to be significantly more informative, i.e. the

Published by Bioscientifica Ltd. 


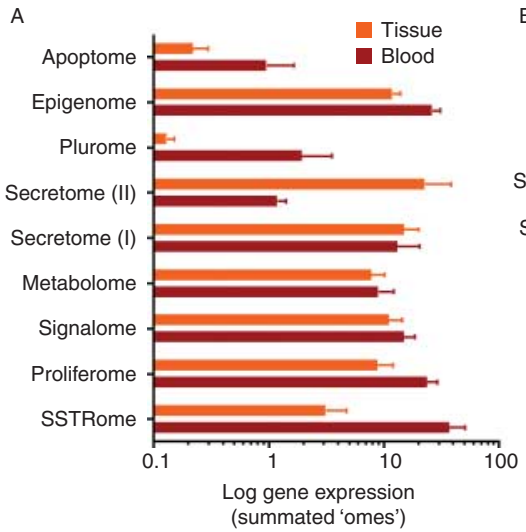

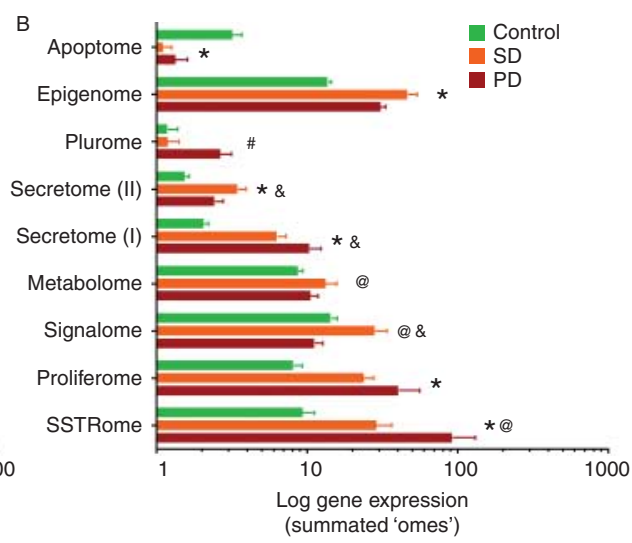

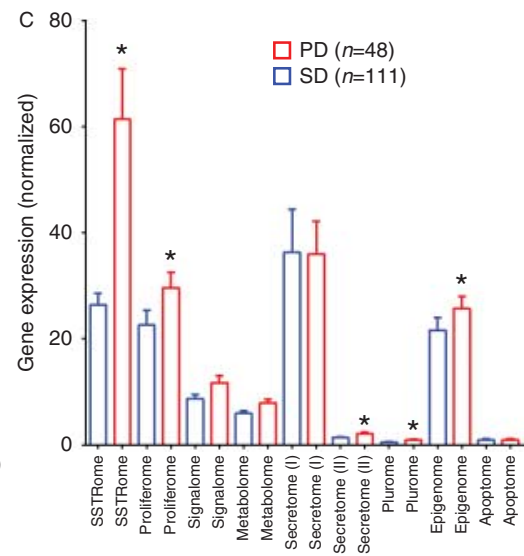

Figure 4

Correlation between marker gene expression as a cluster 'ome' in tumor tissue and in peripheral blood in three different sets. (A) Gene cluster (GC) expression (individual 'omes') in tumor tissue was not significantly different to that in blood ( $P=0.11$ ). (B) GC expression (individual 'omes') in control blood (green: $n=67)$, in stable disease (SD: $n=35)$ and in progressive/active disease $(n=28)$. Significant differences were noted for NETs compared to control blood for the apoptome, epigenome, secretome (I and II),

difference between AUC: $0.04 \pm 0.013, Z$-statistic $=3.139$, $P=0.00017$ (Fig. 6D). Of particular note was that the higher NET scores (6-7), which previously could not accurately differentiate SD vs PD could now be accurately differentiated (93\%) by the activity-based index.

\section{Discussion}

It is clear that monoanalyte biomarkers such as chromogranin A have significant limitations in the diagnosis and management of NETs (Marotta et al. 2012). Having developed an effective multianalyte strategy for NET disease proliferome and SSTRome. SD had higher metabolome and signalome representation while PD had higher plurome expression. Mean \pm s.E.M. ${ }^{*} P<0.05$ vs controls, ${ }^{\#} P<0.05$ vs control and SD, ${ }^{\&} P<0.05$ vs $P D$, and ${ }^{\circledR} P<0.05$ vs control (Mann-Whitney $U$ test, two-tailed). (C) Each of the nine GC 'omes' in the independent set of NETs $(n=159)$. PD was signified by elevated SSTrome, proliferome, secretome (II), plurome and epigenome activities. Mean \pm s.E.M. ${ }^{\star} P<0.05$ vs SD.

diagnosis, we sought to amplify the utility of this diagnostic by incorporating tumor-derived biological and gene network information. Our current investigations assess whether measurements of circulating transcripts directly correlated with tumor tissue expression levels and whether this 'liquid' biopsy could yield further information about the biological map ('hallmarks') of the tumor. Ultimately, we sought to demonstrate that a CIA blood measurement would be able to identify and differentiate clinically SD from PD. Our results demonstrated that circulating transcripts detectable in blood were expressed in and significantly correlated with tumor tissue irrespective of the origin. While

Table 5 Gene clusters, clinical outcome, and ROC-derived information from the different 'omes' for differentiating SD from PD

\begin{tabular}{l} 
Cluster name \\
\hline Proliferome \\
Growth factor signalome \\
Metabolome \\
Secretome I (general) \\
Secretome II (progressive) \\
Epigenome \\
Apoptome \\
Plurome \\
SSTRome
\end{tabular}

\begin{tabular}{|c|c|c|c|}
\hline \multicolumn{4}{|c|}{ Test set $(n=130)$} \\
\hline Kruskal-Wallis ${ }^{\mathrm{b}}$ & Con vs SD & Con vs $\mathrm{PD}^{c}$ & SD vs $\mathrm{PD}^{\mathrm{c}}$ \\
\hline$P<0.0001,51.7$ & $P<0.0001$ & $P<0.0001$ & NS \\
\hline$P=0.0055,10.4$ & $P=0.017$ & NS & $P=0.012$ \\
\hline$P=0.43,1.7$ & NS & NS & NS \\
\hline$P<0.0001,54.1$ & $P<0.0001$ & $P<0.0001$ & NS \\
\hline$P<0.0001,22.7$ & $P<0.0001$ & $P=0.08$ & NS \\
\hline$P<0.0001,51.8$ & $P<0.0001$ & $P<0.0001$ & NS \\
\hline$P=0.011,9.1$ & $P=0.02$ & $P=0.09$ & NS \\
\hline$P<0.0001,33.96$ & & $P=0.0004$ & $P=0.0035$ \\
\hline$P<0.0001,34.0$ & $P=0.0078$ & $P<0.0001$ & $P=0.03$ \\
\hline
\end{tabular}

Combined sets (test + independent) ${ }^{\mathrm{a}}$

\begin{tabular}{llccc}
\hline \multicolumn{1}{c}{ AUC } & & $95 \% \mathrm{Cl}$ & & $P$ value \\
\cline { 1 - 1 } $0.698 \pm 0.035$ & & $0.629-0.767$ & & $<0.0001$ \\
$0.574 \pm 0.04$ & & $0.496-0.652$ & & 0.07 \\
$0.652 \pm 0.04$ & & $0.573-0.730$ & & 0.0002 \\
$0.514 \pm 0.041$ & & $0.434-0.594$ & & 0.73 \\
$0.655 \pm 0.037$ & & $0.583-0.727$ & & 0.0002 \\
$0.721 \pm 0.039$ & & $0.644-0.797$ & & $<0.0001$ \\
$0.70 \pm 0.034$ & & $0.636-0.771$ & & $<0.0001$ \\
$0.654 \pm 0.038$ & & $0.580-0.729$ & & 0.0002 \\
$0.683 \pm 0.038$ & & $0.610-0.759$ & & $<0.0001$ \\
& & &
\end{tabular}

AUC, area under the curve; NS, not significant.

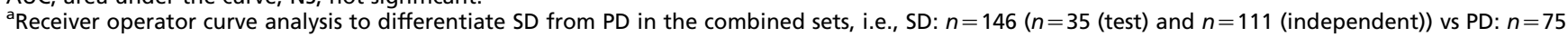
$(n=27$ (test) and $n=48$ (independent)).

${ }^{\mathrm{b}}$ Kruskal-Wallis test including $P$ value and the KW statistic.

'Dunn's multiple comparison test $(\alpha<0.05)$. Multiplicity adjusted $P$ values are reported. 

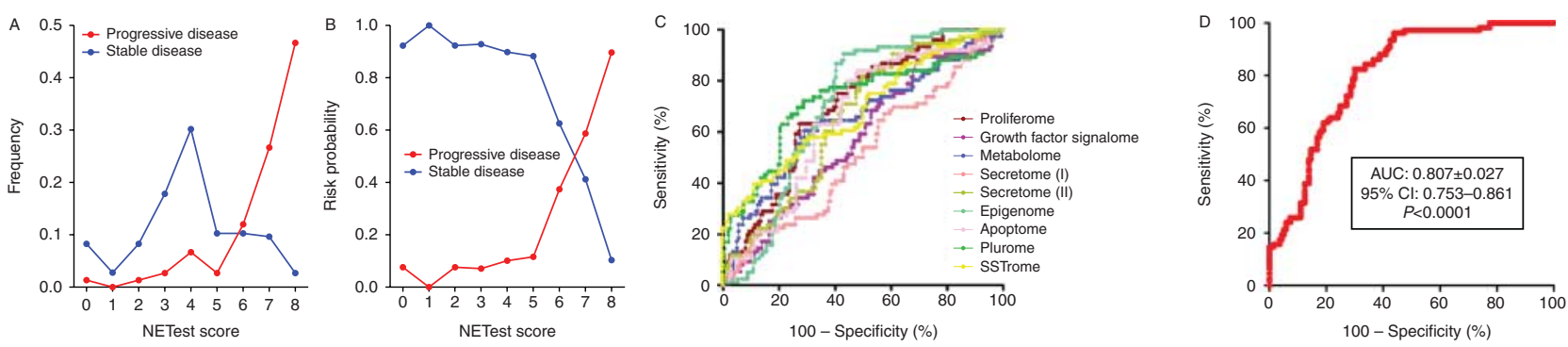

Figure 5

Assessment of NET score and 'omes' in peripheral blood. ( $\mathrm{A}$ and $\mathrm{B}$ ) Frequency distribution for the $0-8$ score in stable disease and in progressive disease $(P D)$ in the combined sets $(A)$ and risk probability analysis for a score being either stable or progressive (B). Scores of 6-7 correlate poorly with disease. (C) ROC curves for each of the 'omes' for differentiating between

the number of tumor samples from individual sites was limited, the distribution is broadly representative of the populations typically seen in clinical practice. Biologically relevant hallmarks (GC/omes) were also identified and shown to have concordance between blood and tumor tissue. The integration of this information with the original MAAA (NET score 0-8) enabled the development of a more

A

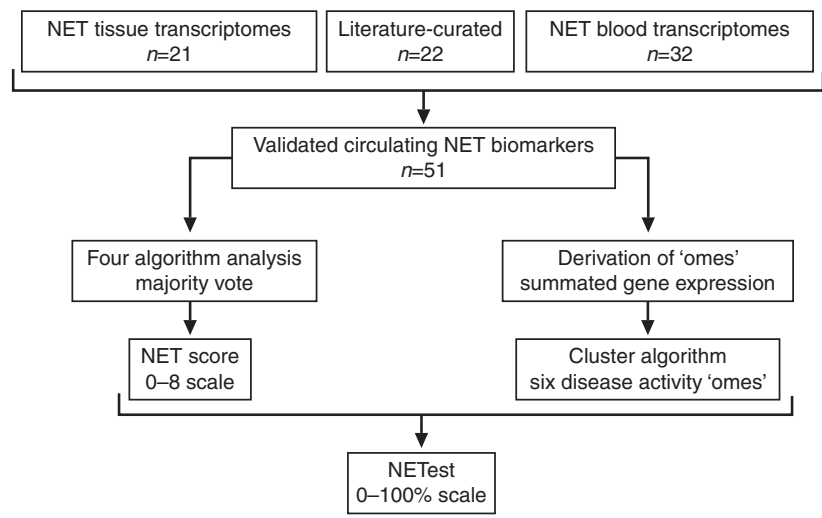

SD and PD in the combined sets. These ranged from AUC of 0.51 (secretome (I)) to 0.72 (plurome). Individual 'omes' are identifiable by different colors. (D) Efficacy of a combined score (including SSTRome, proliferome, epigenome, metabolome, secretome (II), and plurome). The AUC for differentiating SD from PD was $0.81, P<0.0001$.

sophisticated tool, a disease activity scale (0-100\%) called the NETest. This numeric exhibits a high sensitivity and specificity (91 and 85\% respectively) for delineating GEP-NET disease activity. The demonstration that this signature is also applicable to bronchopulmonary NETs, suggests its likely utility in the evaluation of these tumors but this requires further evaluation.

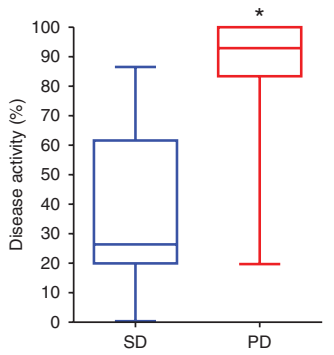

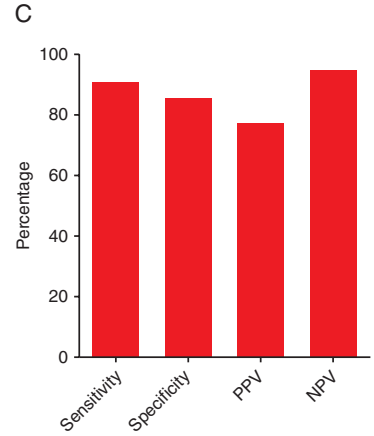

D

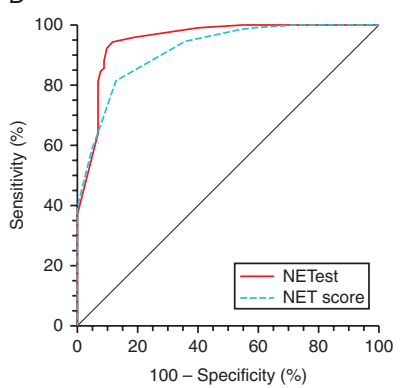

\section{Figure 6}

Development and assessment of disease activity NET score - the NETest - in stable disease and progressive disease (PD) in peripheral blood (combined data set: $n=222$ ). (A) Graphic demonstrating the development of the NET score and cluster identification with the resultant NETest and 0-100 scale of disease activity. (B) Distribution (box and whisker - minimum to maximum, medians) of the NETest (combination of NET score and cluster information)
(C) 2015 Society for Endocrinology Printed in Great Britain in stable disease (SD) and PD. Values were significantly different. $\star P<0.0001$ vs SD. (C) Performance metrics of the NETest for differentiating SD from PD in the combined cohort. Metrics were $>80 \%$. (D) ROC curves for the NETest (red line) and the NET score (blue line). The NETest was associated with a significantly better AUC than the NET score $(0.92 \pm 0.02$ vs $0.88 \pm 0.02$, difference: $0.04 \pm 0.013, Z$-statistic $=3.139, P=0.00017$ ). 
The use of PCR to determine transcript alterations, e.g. gene fusions in acute myeloid leukemia 1 (AML1)/ETO or BCR-ABL in peripheral blood, is now a standard approach in hematological cancers (Oehler \& Radich 2006, Zhang et al. 2014). Similar methodologies are evolving for solid tumors, e.g. MACC1 in gastric cancers (Burock et al. 2015) or a panel of prostate cancer-associated genes in prostate cancer (Danila et al. 2014). Hematological cancer measurements clearly reflect a pathobiological process as expression of fusion transcript levels correlate well $\left(R^{2}=\sim 0.5\right)$ between bone marrow and blood (Ostergaard et al. 2004). However, it is unclear whether measurements of solid tumor-associated transcripts reflect actual carcinoma tissue expression or capture other aspects of tumor biology, e.g. gene expression from non-tumor cells in the tumor microenvironment. We specifically assessed the 51 marker gene expression in NET tumor tissue and identified that levels were elevated in 22 tumors from different GEP sites compared to macroscopically normal mucosa. Ninety-eight percent of transcripts exhibited elevated expression in the tumor samples identifying that transcripts were neuroendocrine-selective. Furthermore, expression of the 51 transcripts could be detected and measured in circulating blood and expression levels exhibited a significant correlation $(R=\sim 0.3, P<0.05)$ with tissue levels irrespective of the origin demonstrating this was a pan-NET signature. Importantly, the NET-derived score in blood was well-correlated (Spearman $r>0.8$, $P<0.0005)$ with tissue. Furthermore, it was completely accurate (100\% samples correctly called as either tumor or normal). These results confirm that circulating transcripts detectable in blood are also expressed in and correlate significantly with GEP-NET tissue expression levels.

We next focused on the 'hallmarks' of NETs. The complexity of cancer has been reduced to a defined number of underlying principles (Hanahan \& Weinberg 2000) which have been recently updated (Hanahan \& Weinberg 2011). This approach has been transposed to NET disease (Walenkamp et al. 2014). Using networking analysis, cancer hallmarks can be signified by molecular networks which have the advantage of allowing quantification and computational modeling (Wang et al. 2014). These networks interact with each other during cancer cell evolution and may thus be considered to define or regulate the evolution of a tumor. Interrogation of such interactions can be quantified and then computationally modeled to predict cancer evolutionary paths and clinical phenotypes. Using this strategy, network operational signatures have been proposed to compute and model the measures of hallmark traits and this systems biology approach identified nine networks (Wang et al. 2014). We applied this stratagem to assess the
51 NET marker genes. Our literature-curated analysis and unbiased protein:protein interactome approach which includes information from all neoplasia identified that thirty of the 51 genes (58\%) could be classified into nine hallmarks or GC network signatures (Table 3). The remainder were not categorized as cancer-relevant hallmarks based on our current understanding of neoplasia. Some of these genes have been identified in NETs, e.g. Ki67 and nucleosome assembly protein-like 1 (NAP1L1) as markers of proliferation (Kidd et al. 2006, Rindi \& Wiedenmann 2011) and mechanistically linked to the epigenome (Schimmack et al. 2014). Growth factor expression and signaling is a well-known regulator of NET proliferation (Kidd et al. 2005) and signaling pathways (e.g. the RAS/RAF/MAPK signaling pathway) is typically activated (Perren et al. 2004, Tannapfel et al. 2005) while expression of the BRAF activator Rap1 as well as B-Raf itself can be detected by immunohistochemistry (75\%) (Karhoff et al. 2007). Other examples include secretion, e.g. PNMA2 as well as somatostatin receptor expression, both of which have been described in NETs (Reubi et al. 2001, Cui et al. 2010).

Initially, we assessed whether the hallmarks or 'omes' exhibited an elevated expression in NET tissue and identified that seven were elevated compared to normal mucosa, with one significantly reduced in tumor tissue compared to normal mucosa (the 'plurome'). We interpreted this observation as evidence that the cancer network-based signatures were selective for NETs compared to normal mucosa, which has been defined as a dynamic, well-regulated tissue with active regulated turnover (Gracz \& Magness 2014). Of clinical relevance, however, was the observation that no significant differences were noted between cluster analysis in matched tissue and blood $(P=0.11)$ although individual 'omes' were generally higher in blood than tissue except for genes involved in secretion (secretome). Differences in transcript expression between tissue and blood are not unusual and have previously been noted in bone marrow diseases where expression levels of certain fusion genes can be three to five times higher in the circulation than in marrow while others may be one to two times lower (Ostergaard et al. 2004). While the mechanism behind these differences is unknown, the higher levels we noted in the circulation were not significantly different to that measured in tumor tissue.

Having determined that the genes could be classified into a cancer network and using the premise that disease activity is linked to cluster expression, we next sought to identify whether GC expression/omes could define clinical disease. We therefore examined the question of

Published by Bioscientifica Ltd. 
whether disease categorized as clinically stable could be differentiated from active, PD by GC/ome expression. The NET specificity of the 'omic' expression was indicated by the elevated expression of the majority, e.g. proliferome, signaling and SSTRome in NETs compared to control blood in the test set. Furthermore, in an independent set ( $n=159)$, the SSTRome, proliferome, secretome, plurome, and epigenome were all significantly increased $(P<0.05)$ in the PD (Fig. 4C). These observations were consistent with our hypothesis that a circulating gene signature captures biologically relevant data that would correlate with clinical and imaging-based assessment of NETs. Measurements of individual 'omes' were individually informative with AUC's ranging from 0.514 (secretome I) to 0.72 (plurome). However, when mathematically combined as a group (into an activity algorithm), the information was highly informative with an AUC of 0.81 for differentiating between PD and clinically SD.

In previous reports, we have detailed the development of a highly accurate diagnostic test for NETs (sensitivity and specificity: 98 and 97\% respectively; Modlin et al. $2013 a, 2014 a, b, c, d)$ based on a $0-8$ score derived from four different prediction algorithms. This investigation sought to evaluate whether the inclusion of the informative clusters (biological data) would amplify the diagnostic potential and provide a predictive component to the assessment of disease activity. To accomplish this, we adopted a weighted approach, similar to that successfully used for measuring/predicting prognosis in non-small cell lung cancer (a five gene and protein signature; Kadara et al. 2011). In our approach, we weighted the $0-8$ score by whether the GC activity algorithm identified the sample as 'active' and therefore 'progressive' or exhibited a low activity gain and was clinically stable. We expressed this coefficient of activity as a $0-100$ score for which the discriminant index was significantly more effective at segregating the two clinical phenotypes. Adoption of this mathematical strategy provided an AUC of 0.92 , with CIA NETest metrics of sensitivity: $91.1 \%$, specificity: $85.3 \%$, positive predictive value: $77.4 \%$, and negative predictive value: $94.6 \%$. This was significantly more accurate than use of the $0-8$ score alone ( $Z$ value: $3.1, P<0.002$ ) and efficiently differentiated individuals with indeterminate scores of 6 and 7 into high probability (93\%) SD or PD.

We developed an accurate blood-based multianalyte transcript diagnostic tool for NETs and now report further refinement by inclusion of 'omic' expression cluster analysis from NET-derived cancer networks. Integration of cluster analysis provides a significantly more effective methodology to facilitate a real-time predictive assessment of NET disease status. In most patients, grading and classification of tumor type is based upon one-time assessment of the primary lesion. This also provides a resource to determine the genetic basis of the disease which may impact treatment as well as behavior. Somatic mutations in genes including MEN1, ATRX, DAXX as well as in mTOR signaling (Leotlela et al. 2003) and in the zinc finger YY1 (Cao et al. 2013) have been identified in pancreatic NETs while inactivation of CDKN1B $\left(P 27^{K I P 1}\right)$ has been noted in small intestinal NETs (Francis et al. 2013). ATRX and DAXX are associated with a reduced time of relapse-free survival and a decrease in survival (Marinoni et al. 2014) while mutations in YY1 are associated with a later onset of tumors (Cao et al. 2013). CDKN1B loss is associated with a decreased survival and reported as an independent factor in poor overall survival (Kim et al. 2014). Protein:protein interactome analyses demonstrate that mutations in these genes that characterize the genetic basis of NET disease can be captured by the circulating signature. The pancreas-restricted mutations are all linked to chromatin remodeling ('epigenome') while MEN1 and CDKN1B are involved in growth factor signaling and proliferation ('signalome' and 'proliferome'). The somatic SNVs identified in small intestinal NETs (Banck et al. 2013) also include genes that impact the different NET 'omes' including FGFR2 ('signalome'), EZH2 ('signalome' and 'proliferome') or actually comprise directly the signature itself, e.g. BRAF. Therefore, the circulating NET signature has the capacity to both identify NET-specific genetic alterations as well as provide information relevant to targetable signaling pathways, e.g. mTOR ('signalome'). This bloodbased multianalyte strategy directly reflects tumor tissue biological activity and provides accurate time-specific information that reflects the status of evolving disease (residual or metastases). It is likely that such information will also reflect the efficacy of any therapeutic intervention and thereby provide valuable clinical information to facilitate management. The early identification of active, progressing disease has obvious clinical implications. In those with residual disease, the identification of increased disease activity or diminished treatment efficacy is clinically of considerable relevance to management. Similarly, clinicians utilizing targeted therapies would benefit from quantification of biologically relevant 'omes', e.g. RAF/RAS signaling or somatostatin receptor expression that provide assurance of the presence of a relevant therapeutic target and demonstration of efficacy. For example, PCR-based molecular monitoring of AML has identified that a lack of decline of transcript levels by less than two logs after chemotherapy is a poor prognostic sign (Jaeger \& Kainz 2003). In chronic myeloid leukemia, PCR-based tests that

Published by Bioscientifica Ltd. 
detect the BCR-ABL chimeric transcript predict relapse in the transplant setting are a strong measure in predicting progression-free survival in imantinib-treated patients (Oehler \& Radich 2006). Trials using the quantitative assessment of BCR-ABL as a surrogate outcome marker will examine if such strategies can reduce both the time and cost of clinical trials (Oehler \& Radich 2006).

We have demonstrated that gene expression, measured in blood, reflected tissue expression, that expression of genes in the NETest captured the biology of NET neoplasia, and that integrating these measurements of circulating gene expression could accurately define clinical status. Based on these data, which indicate that measurement of circulating gene expression is clinically informative, we have built an algorithm - the NETEst - that not only accurately diagnoses GEP-NETs but also provides a measure of their biological activity.

\section{Declaration of interest}

The authors declare that there is no conflict of interest that could be perceived as prejudicing the impartiality of the research reported.

\section{Funding}

This work was supported by Clifton Life Sciences.

\section{References}

Banck MS, Kanwar R, Kulkarni AA, Boora GK, Metge F, Kipp BR, Zhang L, Thorland EC, Minn KT, Tentu R et al. 2013 The genomic landscape of small intestine neuroendocrine tumors. Journal of Clinical Investigation 123 2502-2508. (doi:10.1172/JCI67963)

Boulesteix AL \& Slawski M 2009 Stability and aggregation of ranked gene lists. Briefings in Bioinformatics 10 556-568. (doi:10.1093/bib/bbp034)

Bralten LB, Kloosterhof NK, Gravendeel LA, Sacchetti A, Duijm EJ, Kros JM, van den Bent MJ, Hoogenraad CC, Sillevis Smitt PA \& French PJ 2010 Integrated genomic profiling identifies candidate genes implicated in glioma-genesis and a novel LEO1-SLC12A1 fusion gene. Genes, Chromosomes \& Cancer 49 509-517. (doi:10.1002/gcc.20760)

Burock S, Herrmann P, Wendler I, Niederstrasser M, Wernecke KD \& Stein U 2015 Circulating metastasis associated in colon cancer 1 transcripts in gastric cancer patient plasma as diagnostic and prognostic biomarker. World Journal of Gastroenterology 21 333-341. (doi:10.3748/ wjg.v21.i1.333)

Cao Y, Gao Z, Li L, Jiang X, Shan A, Cai J, Peng Y, Li Y, Jiang X, Huang X et al. 2013 Whole exome sequencing of insulinoma reveals recurrent T372R mutations in YY1. Nature Communications 4 2810. (doi:10.1038/ ncomms3810)

Cui T, Hurtig M, Elgue G, Li SC, Veronesi G, Essaghir A, Demoulin JB, Pelosi G, Alimohammadi M, Oberg K et al. 2010 Paraneoplastic antigen Ma2 autoantibodies as specific blood biomarkers for detection of early recurrence of small intestine neuroendocrine tumors. PLOS ONE 5 e16010. (doi:10.1371/journal.pone.0016010)

Danila DC, Anand A, Schultz N, Heller G, Wan M, Sung CC, Dai C, Khanin R, Fleisher M, Lilja $\mathrm{H}$ et al. 2014 Analytic and clinical validation of a prostate cancer-enhanced messenger RNA detection assay in whole blood as a prognostic biomarker for survival. European Urology 65 1191-1197. (doi:10.1016/j.eururo.2013.07.006)

Drozdov I, Kidd M, Nadler B, Camp RL, Mane SM, Hauso O, Gustafsson BI \& Modlin IM 2009 Predicting neuroendocrine tumor (carcinoid) neoplasia using gene expression profiling and supervised machine learning. Cancer 115 1638-1650. (doi:10.1002/cncr.24180)

Engels CC, Ruberta F, de Kruijf EM, van Pelt GW, Smit VT, Liefers GJ, Matsushima T, Shibayama M, Ishihara H, van de Velde CJ et al. 2013 The prognostic value of apoptotic and proliferative markers in breast cancer. Breast Cancer Research and Treatment 142 323-339. (doi:10.1007/s10549-013-2748-y)

Fleige S, Walf V, Huch S, Prgomet C, Sehm J \& Pfaffl MW 2006 Comparison of relative mRNA quantification models and the impact of RNA integrity in quantitative real-time RT-PCR. Biotechnology Letters $\mathbf{2 8}$ 1610-1613. (doi:10.1007/s10529-006-9127-2)

Franceschini A, Szklarczyk D, Frankild S, Kuhn M, Simonovic M, Roth A, Lin J, Minguez P, Bork P, von Mering C et al. 2013 STRING v9.1: proteinprotein interaction networks, with increased coverage and integration. Nucleic Acids Research 41 D808-D815. (doi:10.1093/nar/gks1094)

Francis JM, Kiezun A, Ramos AH, Serra S, Pedamallu CS, Qian ZR, Banck MS, Kanwar R, Kulkarni AA, Karpathakis A et al. 2013 Somatic mutation of CDKN1B in small intestine neuroendocrine tumors. Nature Genetics $\mathbf{4 5}$ 1483-1486. (doi:10.1038/ng.2821)

Gracz AD \& Magness ST 2014 Defining hierarchies of stemness in the intestine: evidence from biomarkers and regulatory pathways. American Journal of Physiology. Gastrointestinal and Liver Physiology $\mathbf{3 0 7}$ G260-G273. (doi:10.1152/ajpgi.00066.2014)

Grimm M, Schmitt S, Teriete P, Biegner T, Stenzl A, Hennenlotter J, Muhs HJ, Munz A, Nadtotschi T, Konig K et al. 2013 A biomarker based detection and characterization of carcinomas exploiting two fundamental biophysical mechanisms in mammalian cells. BMC Cancer 13 569. (doi:10.1186/1471-2407-13-569)

Hanahan D \& Weinberg RA 2000 The hallmarks of cancer. Cell 100 57-70. (doi:10.1016/S0092-8674(00)81683-9)

Hanahan D \& Weinberg RA 2011 Hallmarks of cancer: the next generation. Cell 144 646-674. (doi:10.1016/j.cell.2011.02.013)

Hanley JA \& McNeil BJ 1982 The meaning and use of the area under a receiver operating characteristic (ROC) curve. Radiology 143 29-36. (doi:10.1148/radiology.143.1.7063747)

Hanley JA \& McNeil BJ 1983 A method of comparing the areas under receiver operating characteristic curves derived from the same cases. Radiology 148 839-843. (doi:10.1148/radiology.148.3.6878708)

Hogarty MD, Norris MD, Davis K, Liu X, Evageliou NF, Hayes CS, Pawel B, Guo R, Zhao H, Sekyere E et al. 2008 ODC1 is a critical determinant of MYCN oncogenesis and a therapeutic target in neuroblastoma. Cancer Research 68 9735-9745. (doi:10.1158/0008-5472.CAN-07-6866)

Irizarry RA, Bolstad BM, Collin F, Cope LM, Hobbs B \& Speed TP 2003 Summaries of Affymetrix GeneChip probe level data. Nucleic Acids Research 31 e15. (doi:10.1093/nar/gng015)

Jaeger U \& Kainz B 2003 Monitoring minimal residual disease in AML: the right time for real time. Annals of Hematology 82 139-147. (doi:10.1007/ s00277-002-0601-1)

Kadara H, Behrens C, Yuan P, Solis L, Liu D, Gu X, Minna JD, Lee JJ, Kim E, Hong WK et al. 2011 A five-gene and corresponding protein signature for stage-I lung adenocarcinoma prognosis. Clinical Cancer Research $\mathbf{1 7}$ 1490-1501. (doi:10.1158/1078-0432.CCR-10-2703)

Karhoff D, Sauer S, Schrader J, Arnold R, Fendrich V, Bartsch DK \& Horsch D 2007 Rap1/B-Raf signaling is activated in neuroendocrine tumors of the digestive tract and Raf kinase inhibition constitutes a putative therapeutic target. Neuroendocrinology 85 45-53. (doi:10.1159/000100508)

Kauffmann A, Gentleman R \& Huber W 2009 arrayQualityMetrics - a bioconductor package for quality assessment of microarray data. Bioinformatics 25 415-416. (doi:10.1093/bioinformatics/btn647)

Kidd M, Eick G, Shapiro MD, Camp RL, Mane SM \& Modlin IM 2005 Microsatellite instability and gene mutations in transforming growth 
factor- $\beta$ type II receptor are absent in small bowel carcinoid tumors. Cancer 103 229-236. (doi:10.1002/cncr.20750)

Kidd M, Modlin IM, Mane SM, Camp RL, Eick G \& Latich I 2006 The role of genetic markers - NAP1L1, MAGE-D2, and MTA1 - in defining smallintestinal carcinoid neoplasia. Annals of Surgical Oncology 13 253-262. (doi:10.1245/ASO.2006.12.011)

Kidd M, Nadler B, Mane S, Eick G, Malfertheiner M, Champaneria M, Pfragner R \& Modlin I 2007 GeneChip, geNorm, and gastrointestinal tumors: novel reference genes for real-time PCR. Physiological Genomics 30 363-370. (doi:10.1152/physiolgenomics.00251.2006)

Kidd M, Modlin IM \& Drozdov I 2014 Gene network-based analysis identifies two potential subtypes of small intestinal neuroendocrine tumors. BMC Genomics 15 595. (doi:10.1186/1471-2164-15-595)

Kim M, Jang HR, Kim JH, Noh SM, Song KS, Cho JS, Jeong HY, Norman JC, Caswell PT, Kang GH et al. 2008 Epigenetic inactivation of protein kinase D1 in gastric cancer and its role in gastric cancer cell migration and invasion. Carcinogenesis 29 629-637. (doi:10.1093/carcin/bgm291)

Kim HS, Lee HS, Nam KH, Choi J \& Kim WH 2014 p27 loss is associated with poor prognosis in gastroenteropancreatic neuroendocrine tumors. Cancer Research and Treatment 46 383-392. (doi:10.4143/crt.2013.102)

Lawrence B, Gustafsson BI, Kidd M, Pavel M, Svejda B \& Modlin IM 2011 The clinical relevance of chromogranin $\mathrm{A}$ as a biomarker for gastroenteropancreatic neuroendocrine tumors. Endocrinology and Metabolism Clinics of North America 40 111-134, viii. (doi:10.1016/j.ecl.2010.12.001)

Leja J, Essaghir A, Essand M, Wester K, Oberg K, Totterman TH, Lloyd R, Vasmatzis G, Demoulin JB \& Giandomenico V 2009 Novel markers for enterochromaffin cells and gastrointestinal neuroendocrine carcinomas. Modern Pathology 22 261-272. (doi:10.1038/modpathol.2008.174)

Leotlela PD, Jauch A, Holtgreve-Grez H \& Thakker RV 2003 Genetics of neuroendocrine and carcinoid tumours. Endocrine-Related Cancer 10 437-450. (doi:10.1677/erc.0.0100437)

Maglott D, Ostell J, Pruitt KD \& Tatusova T 2011 Entrez Gene: gene-centered information at NCBI. Nucleic Acids Research 39 D52-D57. (doi:10.1093/nar/gkq1237)

Marinoni I, Kurrer AS, Vassella E, Dettmer M, Rudolph T, Banz V, Hunger F, Pasquinelli S, Speel EJ \& Perren A 2014 Loss of DAXX and ATRX are associated with chromosome instability and reduced survival of patients with pancreatic neuroendocrine tumors. Gastroenterology 146 453-460 (e455). (doi:10.1053/j.gastro.2013.10.020)

Marotta V, Nuzzo V, Ferrara T, Zuccoli A, Masone M, Nocerino L, Del Prete M, Marciello F, Ramundo V, Lombardi G et al. 2012 Limitations of chromogranin A in clinical practice. Biomarkers 17 186-191. (doi:10.3109/1354750X.2012.654511)

McClintick JN \& Edenberg HJ 2006 Effects of filtering by present call on analysis of microarray experiments. BMC Bioinformatics 749 . (doi:10.1186/1471-2105-7-49)

Miretti S, Roato I, Taulli R, Ponzetto C, Cilli M, Olivero M, Di Renzo MF, Godio L, Albini A, Buracco P et al. 2008 A mouse model of pulmonary metastasis from spontaneous osteosarcoma monitored in vivo by luciferase imaging. PLoS ONE 3 e1828. (doi:10.1371/journal.pone.0001828)

Modlin I, Drozdov I \& Kidd M 2013a The Identification of gut neuroendocrine tumor disease by multiple synchronous transcript analysis in blood. PLoS ONE 8 e63364. (doi:10.1371/journal.pone.0063364)

Modlin I, Drozdov I \& Kidd M 2013b A multitranscript blood neuroendocrine tumor molecular signature to identify treatment efficacy and disease progress. Journal of Clinical Oncology 31 (Suppl) A4137.

Modlin I, Drozdov I, Alaimo D, Callahan S, Teixeira N, Bodei L \& Kidd M 2014a A multianalyte PCR blood test outperforms single analyte ELISAs for neuroendocrine tumor detection. Endocrine-Related Cancer 21 615-628. (doi:10.1530/ERC-14-0190)

Modlin I, Kidd M, Taylor A, Drozdov I \& Bodei L 2014 $b$ Neuroendocrine tumor biomarkers: current status and perspectives. Neuroendocrinology 100 265-277. (doi:10.1159/000368363)

Modlin I, Drozdov I \& Kidd M 2014c Gut neuroendocrine tumor blood qPCR fingerprint assay: characteristics and reproducibility. Clinical Chemistry 52 419-429. (doi:10.1515/cclm-2013-0496)
Modlin IM, Aslanian H, Bodei L, Drozdov I \& Kidd M 2014d A PCR blood test outperforms chromogranin A in carcinoid detection and is unaffected by PPIs. Endocrine Connections 3 215-223. (doi:10.1530/EC-14-0100)

Muscarella LA, D'Alessandro V, la Torre A, Copetti M, De Cata A, Parrella P, Sperandeo M, Pellegrini F, Frusciante V, Maiello E et al. 2011 Gene expression of somatostatin receptor subtypes SSTR2a, SSTR3 and SSTR5 in peripheral blood of neuroendocrine lung cancer affected patients. Cellular Oncology 19 19. (doi:10.1007/s13402-011-0025-9)

Naik S, Dothager RS, Marasa J, Lewis CL \& Piwnica-Worms D 2009 Vascular endothelial growth factor receptor-1 is synthetic lethal to aberrant $\beta$-catenin activation in colon cancer. Clinical Cancer Research $\mathbf{1 5}$ 7529-7537. (doi:10.1158/1078-0432.CCR-09-0336)

Oehler VG \& Radich JP 2006 Monitoring BCR-ABL in the treatment of chronic myeloid leukemia by polymerase chain reaction. Current Hematologic Malignancy Reports 1 152-159. (doi:10.1007/s11899-996-0003-x)

Ostergaard M, Olesen LH, Hasle H, Kjeldsen E \& Hokland P 2004 WT1 gene expression: an excellent tool for monitoring minimal residual disease in 70\% of acute myeloid leukaemia patients - results from a singlecentre study. British Journal of Haematology 125 590-600. (doi:10.1111/ j.1365-2141.2004.04952.x)

Perren A, Schmid S, Locher T, Saremaslani P, Bonvin C, Heitz PU \& Komminoth P 2004 BRAF and endocrine tumors: mutations are frequent in papillary thyroid carcinomas, rare in endocrine tumors of the gastrointestinal tract and not detected in other endocrine tumors. Endocrine-Related Cancer 11 855-860. (doi:10.1677/erc.1.00841)

Raza A, Ali Z, Irfan J, Murtaza S \& Shakeel S 2012 Analytical variables influencing the HCV RNA determination by TaqMan real-time PCR in routine clinical laboratory practice. Molecular Biology Reports 39 7421-7427. (doi:10.1007/s11033-012-1574-3)

Reubi JC, Waser B, Schaer JC \& Laissue JA 2001 Somatostatin receptor sst1-sst5 expression in normal and neoplastic human tissues using receptor autoradiography with subtype-selective ligands. European Journal of Nuclear Medicine 28 836-846. (doi:10.1007/s002590100541)

Rindi G \& Wiedenmann B 2011 Neuroendocrine neoplasms of the gut and pancreas: new insights. Nature Reviews. Endocrinology 2120. (doi:10.1038/nrendo.2011.120)

Rindi G, Kloppel G, Alhman H, Caplin M, Couvelard A, de Herder WW, Erikssson B, Falchetti A, Falconi M, Komminoth P et al. 2006 TNM staging of foregut (neuro)endocrine tumors: a consensus proposal including a grading system. Virchows Archiv 449 395-401.

Rindi G, Kloppel G, Couvelard A, Komminoth P, Korner M, Lopes JM, McNicol AM, Nilsson O, Perren A, Scarpa A et al. 2007 TNM staging of midgut and hindgut (neuro) endocrine tumors: a consensus proposal including a grading system. Virchows Archiv 451 757-762.

Schimmack S, Taylor A, Lawrence B, Alaimo D, Schmitz-Winnenthal H, Buchler MW, Modlin IM \& Kidd MA 2014 A mechanistic role for the chromatin modulator, NAP1L1, in pancreatic neuroendocrine neoplasm proliferation and metastases. Epigenetics \& Chromatin 715. (doi:10.1186/1756-8935-7-15)

Tannapfel A, Vomschloss S, Karhoff D, Markwarth A, Hengge UR, Wittekind C, Arnold R \& Horsch D 2005 BRAF gene mutations are rare events in gastroenteropancreatic neuroendocrine tumors. American Journal of Clinical Pathology 123 256-260. (doi:10.1309/ YQBR9C05RU4DD3RN)

Travis WD, Brambilla E, Muller-Hermlink H \& Harris C 2004 World Health Organization classification of tumours. Pathology and genetics of tumours of the lung, pleura, thymus and heart. Lyon, France: IARC Press.

Walenkamp A, Crespo G, Fierro Maya F, Fossmark R, Igaz P, Rinke A, Tamagno G, Vitale G, Oberg K \& Meyer T 2014 Hallmarks of gastrointestinal neuroendocrine tumours: implications for treatment. Endocrine-Related Cancer 21 R445-R460. (doi:10.1530/ERC-14-0106)

Wang E, Zaman N, McGee S, Milanese JS, Masoudi-Nejad A \& O'ConnorMcCourt M 2014 Predictive genomics: a cancer hallmark network framework for predicting tumor clinical phenotypes using genome sequencing data. Seminars in Cancer Biology 30 4-12. (doi:10.1016/j. semcancer.2014.04.002) 
Wulbrand U, Wied M, Zofel P, Goke B, Arnold R \& Fehmann H 1998 Growth factor receptor expression in human gastroenteropancreatic neuroendocrine tumours. European Journal of Clinical Investigation 28 1038-1049. (doi:10.1046/j.1365-2362.1998.00397.x)
Zhang L, Cao Z, Ruan M, Zeng Q, Zhao L, Li Q, Zou Y, Wang J \& Zhu X 2014 Monitoring the AML1/ETO fusion transcript to predict outcome in childhood acute myeloid leukemia. Pediatric Blood \& Cancer 61 1761-1766. (doi:10.1002/pbc.25109)

Received in final form 31 May 2015

Accepted 2 June 2015

Made available online as an Accepted Preprint

2 June 2015
Published by Bioscientifica Ltd. 